\title{
Hue-based quantification of mechanochromism towards a cost-effective detection of mechanical strain in polymer systems
}

\author{
A. Battisti, ${ }^{a, b}$ P. Minei, ${ }^{c}$ A. Puccic,* and R. Bizzarria, ${ }^{a, b}, *$
}

HSV is a digital colour space easily accessible by transformation of RGB images. In this work, the hue parameter $\mathrm{H}$ was used to assess mechanically-induced colour changes of the aggregation-sensitive fluorescent dye 4,4'-bis-(2-benzoxazolyl) stilbene (BBS), thus implementing a cheap and reliable method for the detection of mechanical deformation in a polymer matrix.

Colorimetric sensors are widely employed in the detection and quantification of analytes such as gases, $, 0,0$ anions, ${ }^{0,0}$ cations, $, 0,0$ protons, ${ }^{0}$ biomolecules, $, 0,0$ but also in the detection of other stimuli such as deformation, ${ }^{0}$ temperature alterations,${ }^{0,0}$ light exposure, $, 0,0$ etc. The basic principle underlying the use of such sensors is the predictable relationship between the presence of the relevant analyte or stimulus and changes in the wavelength of light absorbed and/or emitted by the sensing molecule.

Among colorimetric fluorescent sensors, it is worth citing the fluorescent dye 4,4'-bis-(2-benzoxazolyl) stilbene (BBS, Scheme 1). BBS is a stilbene derivative typically employed as an optical brightener in several polymers thanks to its high compatibility with the continuous matrices. ${ }^{0}$ Moreover, the high resistance of this fluorophore to solvent extraction and its very high melting point $\left(360^{\circ} \mathrm{C}\right)$ and

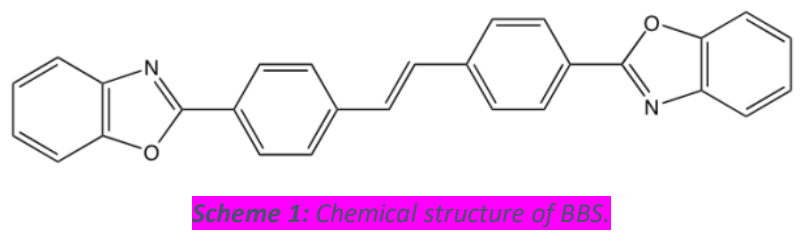

degradation temperature $\left(380^{\circ} \mathrm{C}\right.$ ) comply with the US Food and Drug Administration (FDA) regulations, making it ideal as an additive for food packaging materials. ${ }^{0}$ Its fluorescence response depends on the presence of isolated chromophores, which emit in the blue region, or aggregates accounting for the formation of an excimer-type emission band centred in the green region; ${ }^{0,0}$ the deformation of BBS-

\footnotetext{
a. NEST - Scuola Normale Superiore, Istituto Nanoscienze - CNR (CNR-NANO), p.zza San Silvestro 12, 56127 Pisa (Italy).

b. Consorzio Interuniversitario Nazionale per la Scienza e Tecnologia dei Materiali INSTM, p.zza San Silvestro 12, 56127 Pisa (Italy).

. Dipartimento di Chimica e Chimica Industriale, Università di Pisa, via Moruzzi 13, 56124 Pisa (Italy).

* Corresponding author.

E-mail addresses: andrea.pucci@unipi.it, ranieri.bizzarri@nano.cnr.it

‡ Electronic Supplementary Information (ESI) available: 1. Conversion from RGB to

HSV colour space; 2 . Spectral centre of gravity (CG) calculation; 3. Preparation of

BBS/LLDPE films; 4. Preparation, spectroscopic characterization and imaging of the

stretched BBS/LLDPE films; 5 . Confocal fluorescence microscopy.
} 
loaded polymeric films (which induces disassembly of BBS aggregates) can then be followed by monitoring the fluorescence colour change, which makes BBS an excellent sensor for smart-material applications. The first example of aggregachromic dyes dispersed in a polymer matrix was reported by Weder et al. who paved

Fig. 1 a: Cylindrical representation of HSV colour space coordinates. $\boldsymbol{b}$ : Correspondence between the HSV values and the emission spectra of two different light sources with same hue $(H)$ and value $(V)$ but different saturation the way for the realization of mechanochromic films based on melt-processed polymer mixtures. ${ }^{0}$ The dispersion of a dye into a polymer is commonly employed in commodity plastics and the procedure has been used for pigmented materials since the early days of the plastic era. ${ }^{0}$ The chances to exploit the features of mechanochromism are based on the availability of fast, sensitive and cost-effective detecting techniques. In this context, we reasoned that the current widespread use of digital devices equipped with RGB cameras might provide a low-cost
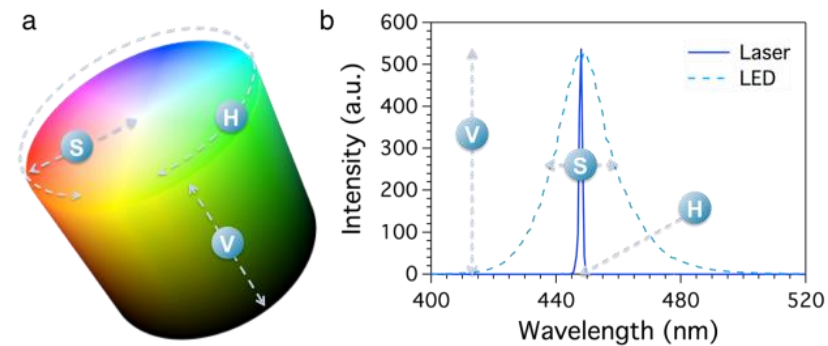

alternative to conventional wavelength-discriminating instruments relying on the sequential use of optical discriminators such as filters and monochromators. Accordingly, we disclose a simple and inexpensive method for the detection of colour changes in the fluorescence of BBS-loaded polymers upon deformation. Remarkably, this approach is not limited to BBS but it could be applied to a wide range of colorimetric systems, spanning from material science to industrial quality control.

Recently, Hakonen et al. demonstrated the possible use of HSV (Hue, Saturation and Value) colour space to monitor colour changes in a $\mathrm{pH}$-sensitive sensor. ${ }^{0}$ Indeed, digital images can be converted to several formats depending on the kind of information that has to be collected. HSV (also known as HSB, Hue Saturation and Brightness) is a digital colour format where the colour of each pixel is identified by the coordinates of its position in a cylindrical space (Fig. 1a). The three different coordinates are: hue $(H)$, that represents the colour tone expressed as an angular value from $0^{\circ}$ to $360^{\circ}$ around the cylinder circumference, saturation (S), that ranges from $100 \%$ (pure colour) to $0 \%$ (grey) and locates the point along the cylinder radius, and value (V), that consists in the maximum intensity of the signal given by the pixel, locating the pixel along the cylinder axis. The mathematical piecewise equations used to obtain the HSV values from a RGB image are reported in the ESI. ${ }^{\ddagger}$ It is worth noting that neutral colours (greys) are undefined in this space; actually, the grey scale is given by any colour showing a $0 \%$ value for $\mathrm{S}$ and a $\checkmark$ ranging from $0 \%$ (black) to $100 \%$ (white). ${ }^{0}$

Remarkably, the HSV coordinates are intimately connected with the physical properties of collected light. Considering the light spectrum, $\mathrm{H}$ reflects the emission wavelength, $\mathrm{S}$ the bandwidth of the peak and $\mathrm{V}$ its height. Fig. $1 \mathrm{~b}$ shows the emission spectra of two different blue light sources, i.e. a laser source and a LED lamp. In this example, the two sources show same emission intensity $(\mathrm{V})$ and wavelength $(\mathrm{H})$ but different peak width (S); the laser light emission profile (solid line) shows a very narrow band, corresponding to a nearly pure colour, while the LED lamp (dashed line) produces a broad band, reflecting a lower saturation value.

Cantrell et al. demonstrated how RGB images collected with any digital device can be easily converted to the relevant HSV stack and compared in terms of colour hue $\mathrm{H}$ without artefacts due to different illumination conditions, which would affect only the $\mathrm{V}$ and $\mathrm{S}$ parameters. ${ }^{0}$ Accordingly, the emission wavelength of a colorimetric fluorescent sensor is meant to be precisely identified by a given hue value, 
and this feature has been exploited in the sensing of $\mathrm{pH}^{0}{ }^{0}$ ions,, 0 and to monitor the degradation of porous silicon photonic crystals. ${ }^{0}$

Following this approach, BBS was homogeneously dispersed in a linear low-density polyethylene matrix (LLDPE). LLDPE is a mostly linear polymer that differs from low-density polyethylene (LDPE) in the absence of long side chains. LLDPE shows higher resistance to impact and puncture and it easily extends under stress. It finds broad application in the production of thin films for stretch cling sheets, plastic bags, silage films, plastic wraps. LLDPE films containing $0.3 \mathrm{wt} \%$ of BBS were prepared by compression moulding of the relevant dye/polymer mixtures, obtained by melt blending the components in a discontinuous Brabender type mixer. After removal from the press, the BBS/LLDPE films were allowed to cool down to room temperature (see $\mathrm{ESI}^{\ddagger}$ for further details). The films were then cut in pieces of $1 \times 1 \mathrm{~cm}$ and stretched according to definite stretching ratios from 1:1 (not stretched) to 6:1 (i.e. six times the original length). Stretching ratios were calculated as the ratio between the final and the original distance separating two ink-marks printed on the films before stretching. The films were photochemically characterized by fluorescence spectroscopy ${ }^{\ddagger}$ as reported in Fig. 2 a.

To evaluate the possibility to follow the stretching process by means of hue variations in the HSV colour space, digital images of the samples were taken under UV irradiation at $366 \mathrm{~nm}$ using the on-board camera of a Nokia Lumia 635 mobile phone (see details in the ESI ${ }^{\ddagger}$ ). Pictures were imported in open access software ImageJ (National Institute of Health, Bethesda, MD, USA; available for download at https://imagej.nih.gov/ij/) and converted to HSV colour space by means of the embedded native plugin, thus obtaining a stack of three layers $(H, S$ and $V)$ for each picture. Average $H$ values were obtained from selected areas of the $\mathrm{H}$ layer and rescaled to span the $0-360^{\circ}$ range. The $\mathrm{S}$ and $\mathrm{V}$ layers were used to evaluate the homogeneity of the picture and to select the region of interest (ROI).

The system was first calibrated by taking pictures of the light cast by three reference laser sources (red, green and blue) emitting at 652,532 and $448 \mathrm{~nm}$ respectively. The conversion to HSV gave average hue values of $0.24,134$ and $238^{\circ}$; data were fitted obtaining a good linearity between hue and wavelength (Fig. 3a). Pictures of the stretched polymeric films were then taken under UV irradiation at $366 \mathrm{~nm}$; confocal fluorescence imaging ${ }^{\ddagger}$ was performed on the same samples to monitor changes in their microstructure. Fig. 4 shows the comparison between the original RGB images (top row), the hue layer relevant to the same ROI after the conversion to HSV (middle row) and images collected by confocal fluorescence microscopy (bottom row). The first sample (not stretched) shows an average hue value of about $170^{\circ}$; this value linearly increases with stretching, reaching a plateau around $216^{\circ}$ after a four-
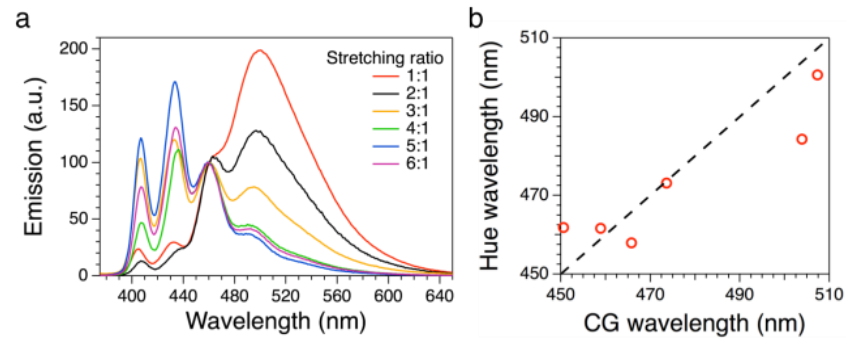

time stretching ratio (Fig. 3b). This behaviour is consistent with spectroscopic data, since the main peak Fig. 2 a: Fluorescence spectra of the BBS/LLDPE films with different stretching
ratios $\left(\lambda_{\text {exc }}=360 \mathrm{~nm}\right)$. b: Wavelength calculated from the hue calibration line
vs CG wavelength.

found for the untreated sample, centred at $500 \mathrm{~nm}$, decreases with stretching, along with the upraising of the two peaks in the blue region (Fig. 2a). We note that the emission spectra of the samples are complex, resulting from the overlapping of several peaks; to visualize a correspondence between the hue and the emission spectra, the spectral centre of gravity (CG, for calculation see $\mathrm{ESI}^{\ddagger}$ ) was plotted against the wavelength corresponding to each hue value according to the calibration line, and a good 
a

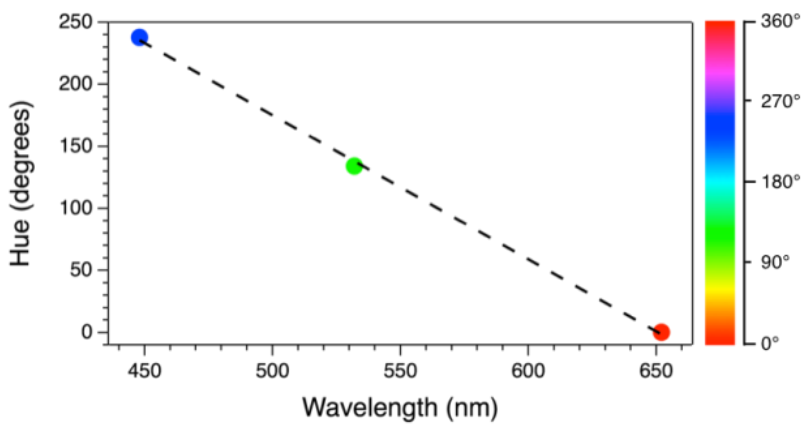

b

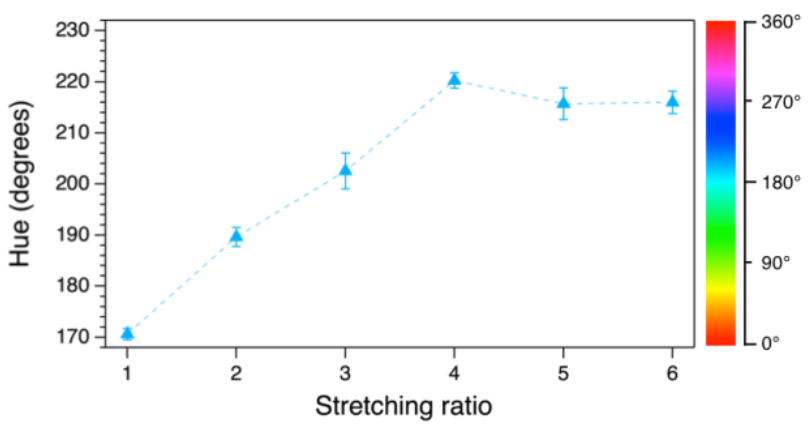

agreement between the two wavelengths was observed as expected (Fig. 2b). It is worth mentioning

Fig. 3 a: Calibration line (dashed) obtained by linearly fitting the reference

lasers hue vs emission wavelength. b: Hue vs stretching ratio of the BBS/LLDPE

films.

that, despite the complexity of the spectra that span from 400 (violet) to almost $600 \mathrm{~nm}$ (red),

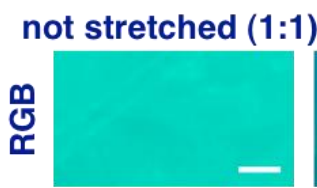

2:1

3:1

$4: 1$
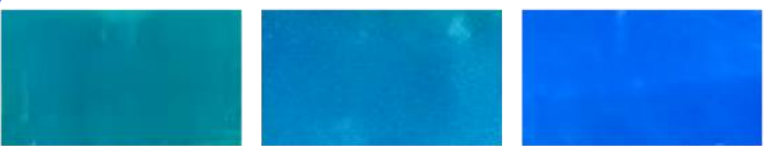

$5: 1$
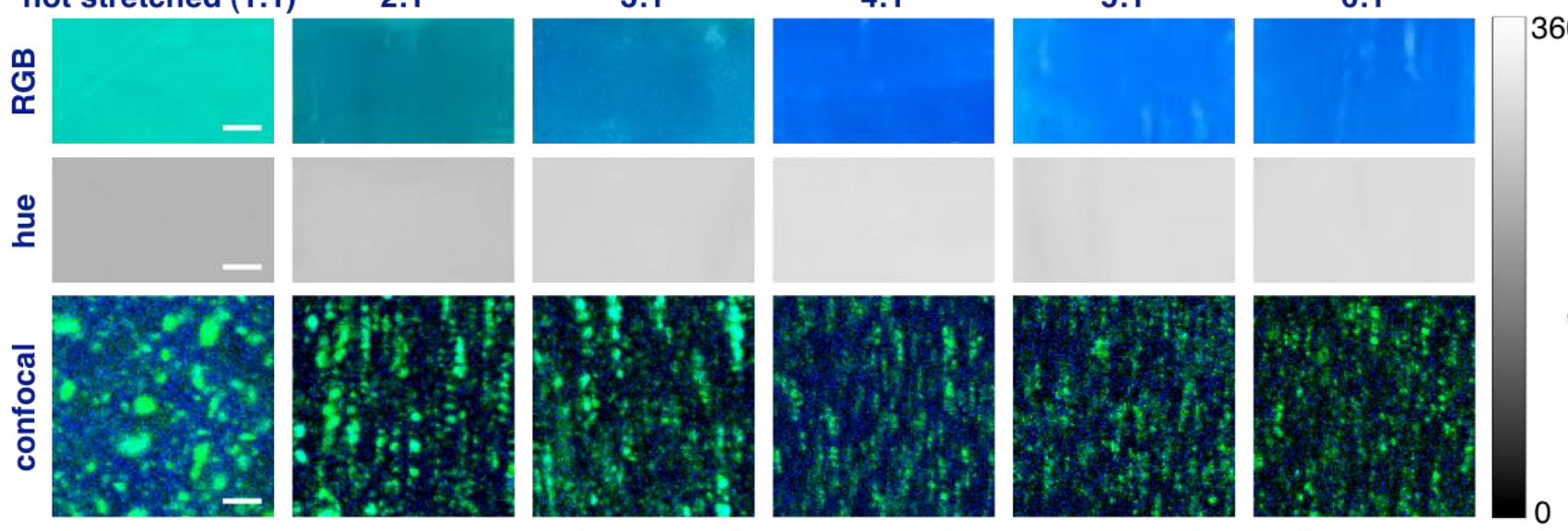

monotonic changes in the position of the centre of gravity can be precisely followed thanks to the hue method. This keeps true for those cases where many emission wavelengths are present, provided that

Fig. 4: Images of BBS/LLDPE samples with different stretching ratios. Top: original RGB images (scale bar = $1 \mathrm{~mm}$ ). Middle: hue layer after the conversion to HSV (scale bar $=1 \mathrm{~mm}$ ). Bottom: confocal fluorescence microscopy images (scale bar $=10 \mu \mathrm{m}$ ). Grey scale bar on the right refers to images in the middle row only. The stretching direction is shown by the red arrow. 
the process under investigation causes a monotonic shift in the emission CG. Limits arise when multiple variations occurring simultaneously compensate each other to some extent; colour alteration in this case could be undistinguishable or not directly relatable to the monitored process due to unpredictable spectral composition.

Notably, colour changes in the samples reflect alterations in the microscopic structure of BBS/LLDPE films due to stretching, as shown by confocal images in the bottom row of Fig. 4: along with the increase in the stretching ratio, the larger BBS clusters are disrupted while smaller elongated aggregates appear, preferentially oriented along the stretching direction. Since this effect is visible only at the microscale, images taken by the camera turn out to be homogeneous, especially when the hue layer is extracted (Fig. 4, middle row); indeed, average light intensity is collected from fairly large areas of the films. The progressive disappearance of large green-emitting aggregates in favour of the blue-emitting monomer accounts for the emission spectrum modification and for the resulting colour change.

In conclusion, the hue parameter allows for extraction of spectral information from digital colour images without the need for wavelength discriminators. Hue evaluation on polymeric films loaded with the mechanochromic dye BBS easily returns the degree of mechanical strain in the polymer. This approach turns out to be valuable as a cost-effective and sensitive tool for several applications such as smart and intelligent packaging, anti-counterfeiting, and quality control systems. We may also envision mobile applications able to calculate in real-time the average hue value of the sample visualized on the device screen by the integrated camera.

\section{Notes and references}

N. A. Rakow and K. S. Suslick, Nature, 2000, 406, 710;

P. Minei, M. Koenig, A. Battisti, M. Ahmad, V. Barone, T. Torres, D. M. Guldi, G. Brancato, G. Bottari and A. Pucci, Journal of Materials Chemistry C, 2014, 2, 9224; H. Maeda and P. Anzenbacher, Supramolecular Chemistry, John Wiley \& Sons, Ltd, 2012;

R. M. Duke, E. B. Veale, F. M. Pfeffer, P. E. Kruger and T. Gunnlaugsson, Chemical Society Reviews, 2010, 39, 3936;

N. Kaur and S. Kumar, Tetrahedron, 2011, 67, 9233;

G. Chen, Z. Guo, G. Zeng and L. Tang, Analyst, 2015, 140, 5400;

G. Liebsch, I. Klimant, C. Krause and O. S. Wolfbeis, Analytical Chemistry, 2001, 73, 4354;

M. J. Antony and M. Jayakannan, Langmuir, 2011, 27, 6268;

Y. Zhou, Z. Xu and J. Yoon, Chemical Society Reviews, 2011, 40, 2222;

F. Ciardelli, G. Ruggeri and A. Pucci, Chemical Society Reviews, 2013, 42, 857;

A. Seeboth, D. Lötzsch, R. Ruhmann and O. Muehling, Chemical Reviews, 2014, 114, 3037;

C. E. Sing, J. Kunzelman and C. Weder, Journal of Materials Chemistry, 2009, 19, 104;

M. Qin, Y. Huang, F. Li and Y. Song, Journal of Materials Chemistry C, 2015, 3, 9265;

P. Minei, A. Battisti, S. Barondi, M. Lessi, F. Bellina, G. Ruggeri and A. Pucci, ACS Macro Letters, 2013, 2, 317;

F. Ercole, T. P. Davis and R. A. Evans, Polymer Chemistry, 2010, 1, 37;

D. A. Jervis, Plastics Additives \& Compounding, 2003, 5, 42;

M. A. Fourati, W. G. Skene, C. G. Bazuin and R. E. Prud'homme, The Journal of Physical Chemistry A, 2013, 117, 836;

A. Pucci, F. Di Cuia, F. Signori and G. Ruggeri, Journal of Materials Chemistry, 2007, 17, 783;

B. R. Crenshaw and C. Weder, Chemistry of Materials, 2003, 15, 4717; 
K. Hunger (ed), Industrial Dyes: Chemistry, Properties, Applications, 2003, Wiley-VCH Verlag GmbH \& Co. KGaA, Weinheim, Germany;

A. Hakonen, J. E. Beves and N. Stromberg, Analyst, 2014, 139, 3524;

A. R. Smith, ACM SIGGRAPH Computer Graphics, 1978, 12, 12;

K. Cantrell, M. M. Erenas, I. de Orbe-Payà and L. F. Capitán-Vallvey, Analytical Chemistry, 2010, 82,

531;

M. M. Erenas, O. Piñeiro, M. C. Pegalajar, M. P. Cuellar, I. de Orbe-Payá and L. F. Capitán-Vallvey, Analitica Chimica Acta, 2011, 694, 128;

M. Ariza-Avidad, A. Nieto, A. Salinas-Castillo, L. F. Capitán-Vallvey, G. M. Miskelly and M. J. Sailor, Nanoscale Research Letters, 2014, 9, 410. 